\title{
National secular trends in ambient air volatile organic compound levels and biomarkers of exposure in the United States
}

\author{
Stacey L. Konkle ${ }^{a, b}$, Kristina M. Zierold ${ }^{c}$, Kira C. Taylor ${ }^{b}$, Daniel W. Riggs ${ }^{a, d}$, Aruni \\ Bhatnagara, ${ }^{*}$ \\ ${ }^{a}$ Christina Lee Brown Envirome Institute, University of Louisville School of Medicine, Louisville, \\ KY, United States

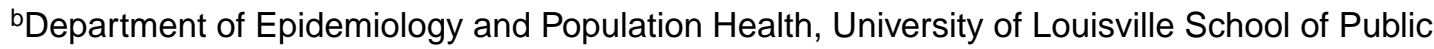 \\ Health and Information Sciences, Louisville, KY, United States \\ 'Department of Environmental Health Sciences, University of Alabama at Birmingham School of \\ Public Health, Birmingham, AL, United States \\ ${ }^{\mathrm{d} D e p a r t m e n t}$ of Bioinformatics and Biostatistics, University of Louisville School of Public Health \\ and Information Sciences, Louisville, KY, United States
}

\section{Abstract}

Background-Exposure to air pollution is a leading cause of global mortality. Volatile organic compounds (VOCs) are constituents of ambient air that could exert adverse health effects.

Objective-To examine the relationship between VOC levels in ambient air and individual-level exposure to VOCs, as assessed by urinary VOC metabolites.

Methods-Secular trends in 11 ambient air VOCs (2005-2013) and individual-level metabolites of 14 VOCs (2005-2014) were assessed using National Monitoring Programs (NMP) and National Health and Nutrition Examination Survey (NHANES) data, respectively. To isolate environmental exposure, individuals reporting exposure to tobacco smoke were excluded. Quantile regression models were used to assess secular trends in VOC exposure, and survey-weighted regression models were built to identify factors associated with VOC exposure.

Results-All annual levels of ambient VOCs decreased from 2005 to 2013 (Range: 12.5\%$77.2 \%)$. However, 11 of the corresponding VOC metabolites increased during the same time (Range: $0.3 \%-53.6 \%$ ). There was a proportional change in patterns of VOC exposure across NHANES waves, with the middle quantiles of exposure showing the largest increase. VOC exposures were significantly associated with age, sex, race, education, and physical inactivity, but not with secular VOC trends.

\footnotetext{
*Corresponding author. Christina Lee Brown Envirome Institute, University of Louisville School, 580 South Preston Street, Baxter II, Room 421C, Louisville, KY, USA. aruni@ louisville.edu (A. Bhatnagar).

Declaration of competing interests

The authors declare they have no actual or potential competing financial interests.

Appendix A. Supplementary data

Supplementary data to this article can be found online at https://doi.org/10.1016/j.envres.2019.108991.
} 
Discussion-In the United States, individual-level exposure to several VOCs increased between 2005 and 2014 despite a decline in ambient air VOC levels. This inverse relationship suggests that ambient VOCs are not the primary source of VOC exposure, therefore, decreasing ambient VOCs alone may not be sufficient to protect against the adverse health effects associated with VOC exposure.

\section{Keywords}

Volatile organic compounds; VOCs; Air pollution Secular trends Environmental health NHANES; Biomarkers of exposure

\section{Introduction}

Air pollution is the leading environmental risk factor that contributes to the global burden of disease (Landrigan et al., 2018). In 2012, it was estimated that one in every nine global deaths was attributable to air pollution exposure, which corresponds to more than 6.5 million deaths per year (WHO, 2016). More recent estimates suggest that exposure to air pollution may be linked to more than 9 million deaths worldwide per year (Landrigan et al., 2018), suggesting that the contribution of air pollution to global mortality may be increasing. Polluted air contains a variety of constituents, including particulate matter (PM), nitrous oxide, ozone, and sulfates, as well as Volatile Organic Compounds (VOCs), which directly contribute to the formation of secondary organic aerosols, a major component of fine PM ( $\mathcal{2} .5 \mu \mathrm{m}$ in aerodynamic diameter; $\mathrm{PM}_{2.5}$ ), and tropospheric ozone (O3) (Dockery et al., 1993; Jerrett et al., 2009; Jimenez et al., 2009).

Previous epidemiologic cohort studies have shown that levels of $\mathrm{PM}_{2.5}$ in ambient air are significantly associated with all-cause and cardiorespiratory mortality as well cardiovascular disease risk and progression (Brook et al., 2010; Landrigan et al., 2018; Pope et al.,2004). Such associations have also been observed even at concentrations of $\mathrm{PM}_{2.5}$ and $\mathrm{O}_{3}$, below the current United States (U.S.) standards (Di et al., 2017), and adverse health effects of PM seem to vary with the composition of polluted air (Bell et al., 2009; Franklin et al., 2008; Laden et al., 2000; Ostro et al., 2007, 2008; Peng et al., 2009; Zanobetti et al., 2009). The current standard in the U.S. for $\mathrm{O}_{3}$ is 0.070 parts per million (ppm) for the annual fourthhighest daily maximum 8-h concentration, averaged over 3 years and for $\mathrm{PM}_{2.5}$ the standard is $12 \mu \mathrm{g} / \mathrm{m}^{3}$ annual mean (EPA, 2016c). The current World Health Organization standard for $\mathrm{O}_{3}$ is $100 \mu \mathrm{g} / \mathrm{m}^{3} 8-\mathrm{h}$ mean and the standard for $\mathrm{PM}_{2.5}$ is $10 \mu \mathrm{g} / \mathrm{m}^{3}$ annual mean (WHO, 2018).

Although most previous work has found that $\mathrm{PM}_{2.5}$ levels are associated with adverse health outcomes, polluted air contains a variety of gaseous co-pollutants such as ozone and nitrous oxides. In addition, ambient air, particularly in urban locations, contains a wide range of VOCs, such as benzene, acrolein, xylene, and formaldehyde (EPA, 2016a). Extensive work has shown that exposure to VOCs both in animal models and humans is associated with systemic, immunologic, neurologic, reproductive, developmental, genotoxic, and carcinogenic effects (Table S1) (ATSDR, 2017). Moreover, the Agency for Toxic Substances 
and Disease Registry (ATSDR) has ranked several of the VOCs of interest in their Substance Priority List as being among the chemicals of top public health concern (ATSDR, 2017).

VOCs are generated by a wide range of indoor and outdoor sources, although tobacco smoke (Delgado-Saborit et al., 2009; Jain, 2015) and automobile emissions (Harley et al., 1997) represent the most frequent sources of exposure. Recent efforts to limit motor vehicle emissions have resulted in a steady decline in ambient VOCs in the U.S. (McCarthy et al., 2007; McDonald et al., 2013); however, the effect of this decline on personal exposure to VOCs remains unknown.

The aim of this study is to assess whether trends in individual-level exposure to VOCs are reflective of ambient VOC levels. Therefore, we examined whether time-dependent changes in the ambient levels of VOCs were reflected in personal-level exposures to VOCs. For this analysis, we obtained data on air toxics from the National Monitoring Programs (NMP). Individual-level exposures in participants of the National Health and Nutrition Examination Survey (NHANES) were estimated by the concentration of the urinary metabolites of VOCs (UM-VOCs), which correspond to individual-level exposures to VOCs (Alwis et al., 2012). We hypothesized that levels of UM-VOCs would reflect ambient VOC concentrations. In addition, we also examined how individual demographic characteristics affect VOC exposure.

These novel nationally-representative estimates of personal-level exposure to VOCs derived from individual participants' urinary metabolites of VOCs provide unique temporal and direct insight into trends and patterns of personal exposure to VOCs in the United States. To date, quantitative and nationally representative trends in individual-level exposure to VOCs using urinary biomarkers of exposure have not been reported.

\section{Methods}

\subsection{Data sources}

Exposure to VOCs was estimated by urinary VOC metabolites in individual participants of NHANES (2005-2014). To complement NHANES UM-VOC data, we analyzed ambient VOC concentrations from the 2005, 2006, 2011, 2012, and 2013 NMP annual reports for ambient air monitoring (EPA, 2016b). Data for 2014 were unavailable. The NMP annual reports summarize research conducted by the Office of Air Quality Planning and Standards, of the U.S. Environmental Protection Agency (EPA). The annual reports contain summary and raw data including measures of ambient concentrations of 59 VOCs from approximately 40 EPA air monitors placed throughout the United States.

We analyzed data from the 2005-06, 2011-12, and 2013-14 waves of NHANES, a nationally representative sample of the general non-institutionalized U.S. population (NCHS, 2015). NHANES gathers vital and health statistics for the nation and is conducted by the National Center for Health Statistics (NCHS) at the Centers for Disease Control (CDC). The cross-sectional survey employs a multi-stage, cluster-sampling design in 2-year cycles to ensure nationally-representative samples and the evaluation of trends over time. Survey participants complete extensive face-to-face interviews, medical examinations, and 
laboratory testing. The NCHS Research Ethics Review Board approves the NHANES study protocols, and all participants provide written informed consent.

We used data from the NMP annual reports to report trends in national mean ambient concentrations of eleven VOCs: acrolein, acrylonitrile, benzene, 1,3-butadiene, carbondisulfide, ethylbenzene, propylene oxide, styrene, toluene, vinyl chloride, and xylene. The eleven NMP ambient VOCs assessed were selected to complement urinary VOC metabolite data available from NHANES. Likewise, we assessed NMP national arithmetic mean ambient concentrations reported in 2005, 2006, 2011, 2012, and 2013 to complement the years of NHANES with available UM-VOC data. The NMP ambient air VOC concentrations were measured using canister samples and gas chromatography/mass spectrometry (GC/ MS). The analytic methods and procedures for these measures have been described in detail in the EPA's Compendium Method TO-15 (EPA, 1999).

Through a partnership with the U.S. EPA, NHANES has provided a platform for the continued study of many important environmental influences on health. Three data release cycles, 2005-06, 2011-12, and 2013-14, included a urinary analysis of VOC metabolites in a subset of participants. In these three NHANES waves, 27 VOC metabolites from 18 parent VOCs were measured in spot urine samples.

Measures from a one-half subsample of participants aged 12 and older in the 2005-06 wave $(\mathrm{n}=3516)$ and a one-third subsample of participants aged 6 and older in both the 2011-12 $(\mathrm{n}=2551)$ and 2013-14 $(\mathrm{n}=2724)$ waves were available for analysis. The metabolites were quantified using ultra performance liquid chromatography coupled with electro spray tandem mass spectrometry (UPLC-ESI-MS/MS); these analyses have been described elsewhere in detail (Alwis et al.,2012).

Although 27 UM-VOCs were measured in the three NHANES waves, to avoid bias in estimation and small sample size among those below the limit of detection (LOD), we selected 20 UM-VOCs, representative of 16 parent VOCs, for which at least $20 \%$ of subjects had concentrations above the LOD. Supplemental Table S2 provides the LOD and the percent of participants above LOD for all $27 \mathrm{UM}$-VOCs measured in NHANES. Table 1 reports the parent compounds and metabolites included in our analysis. For the $20 \mathrm{UM}-$ VOCs assessed in this study, UM-VOCs at or below the LOD were imputed as the LOD divided by the square root of two (NCHS, 2018). To account for differences in dilution, UMVOCs were normalized to urinary creatinine concentrations by dividing each metabolite concentration by the individual's urinary creatinine concentration.

\subsection{Exposure assessment analysis}

Population-Urinary metabolites of VOCs represent a multitude of exposure sources. To isolate exposure to ambient VOCs, we removed individuals exposed to tobacco smoke, the predominant source of VOC exposure (Jain, 2015; Wallace et al., 1987). Tobacco smoke exposure was defined as serum cotinine greater than $0.05 \mathrm{ng} / \mathrm{mL}$ or self-reported personal or in-home tobacco use (Benowitz et al., 2009). Our analysis was limited to individuals who were 20 years of age or older, and who had data available for all UM-VOCs. Individuals 
who were pregnant or breastfeeding at the time of sample collection were excluded. After these exclusions, data were available for a total of 2435 participants.

Analysis Methods-Geometric means (GM), standard errors (SE), and interquartile ranges of creatinine-normalized UM-VOC concentrations were calculated separately for the 2005-06, 2011-12, and 2013-14 NHANES waves. Analysis of variance (ANOVA) was used to assess whether average mean VOC concentrations were significantly different between years $(a=0.05)$. Proportional changes in VOC exposures were assessed by calculating the ratio attributable to each UM-VOC as a proportion of all significantly changing UM-VOCs for each NHANES wave.

A cumulative VOC exposure score was generated to aid in the identification of factors associated with secular trends in aggregate VOC exposures. For each parent VOC represented in NHANES, a representative metabolite was selected based on a significant mean difference across survey years (2005-06, 2011-12, and 2013-14). If a parent VOC had multiple metabolites with significant secular differences across years, the metabolite with the most significant secular difference was selected as the representative metabolite for that VOC. The 11 representative UM-VOCs were categorized into quintiles using cutoff points of 20th, 40th, 60th, and 80th percentile values. UM-VOCs at or below LOD were forced into the lowest exposure group, the 20th percentile group.

To generate a cumulative measure of the significantly different VOCs, we summed the ranks of the representative UM-VOCs. The summary values were subsequently treated as a new continuous exposure measure with a range of 1-55. The cumulative VOC exposure score was then used in survey-weighted regression models as the dependent variable to assess associations with cumulative VOC exposure and demographic, lifestyle, and potential exposure factors. In addition, we analyzed the cumulative VOC exposure models for interaction with NHANES wave to determine whether the association between these factors and VOC levels varied by year. Quantile regression models were used to assess secular changes in the distribution of the cumulative VOC exposure scores. Quantile regression models estimate changes in cumulative VOC exposure score conditional quantiles with changes in NHANES cohort. In addition to the cumulative VOC exposure score, we also examined the associations between individual VOCs and demographic and lifestyle factors. We fitted survey-weighted regression models for individual creatinine-normalized log transformed UM-VOCs to each lifestyle and demographic factor separately.

Additionally, we performed a factor analysis on VOC metabolites to identify underlying sources of exposure. This analysis was performed using log-transformed metabolites with > $40 \%$ of samples above the LOD (16 metabolites).

The mean ambient air VOC concentrations data were analyzed using Excel (Microsoft, Redmond, WA). All other analyses were conducted using SAS software (version 9.4) with the survey package to account for NHANES complex sampling design and weights (SAS, 2018). We found no significant differences in sociodemographic variables between NHANES cohorts, therefore all models were left unadjusted for analysis. Additionally, no adjustment for multiple comparisons was made. 


\section{Results}

We used the levels of 14 UM-VOCs from NHANES data as biomarkers of exposure in survey participants to reflect 11 ambient air VOC concentrations as measured by NMP. All ambient VOC levels decreased from 2005 to 2013 (Range: 12.5\%-77.2\%) (Fig. 1). Eight of the 11 ambient VOC concentrations significantly declined from 2005 to 2013 (Fig. S3). However, all corresponding VOC metabolites increased over approximately the same timeframe (Range: $0.3 \%-53.6 \%$ ) except for one metabolite each from propylene oxide, styrene, and xylene (Fig. 1). Seven of the 14 UM-VOCs significantly increased from the 2005-06 NHANES wave (Fig. S3). The benzene and ethylbenzene/styrene metabolites showed the largest urinary concentration increases.

Five ambient air VOCs significantly decreased, while the six complementary UM-VOCs in NHANES participants increased. The ambient air measurements and personal-level exposures to carbon-disulfide, acrylonitrile, acrolein, ethylbenzene, and benzene showed true significant discordance. Toluene, xylene, and 1,3-butadiene significantly declined in the ambient air from 2005 to 2013, but no significant changes in the complementary NHANES UM-VOC concentrations were observed. On the other hand, HEMA, a metabolite of acrylonitrile, vinyl chloride, and ethylene oxide, significantly increased in NHANES UMVOC concentrations, but no significant changes were observed in ambient air measures.

The discordance in annual ambient VOCs with personal-level exposures measured as UMVOC concentrations in NHANES participants contradicts our original hypothesis that trends in ambient VOCs concentrations would be reflected in personal biomarkers of exposure. Since our original hypothesis was not supported, we further analyzed all available UMVOCs $(\mathrm{N}=20)$ from the cohort of 2435 participants from the 2005-06, 2011-12, and 201314 NHANES waves. The aim of the additional analysis was to improve understanding of the demographic, lifestyle, and potential exposure factors associated with increased VOC exposures in the U.S.

Over the 2005-06, 2011-12, and 2013-14 waves of NHANES, there were no significant changes in basic sociodemographic characteristics (Table 2). The overall cohort had a mean age of 51.6 years, was $55.8 \%$ female, $72.4 \%$ non-Hispanic white, $7.0 \%$ non-Hispanic black, 8.1\% Mexican American, 5.9\% other Hispanic, and 6.6\% other/multi-racial. The cohort was predominantly overweight (57.4\%), had at least a high school education (89.0\%), consumed alcohol (72.6\%), reported being physically active (74.1\%), and made greater than $\$ 20,000$ a year in household income $(89.3 \%)$.

Table 1 shows the distribution of UM-VOCs and p-values associated with changes in all 20 metabolites across the 2005-06, 2011-12, and 2013-14 waves of NHANES. Twelve of the 20 metabolites were significantly different across the years. Of the metabolites with significantly different concentrations across years, all but one, a metabolite of acrylamideGAMA, increased from the 2005-06 NHANES survey. Metabolites of acrylonitrile and carbon-disulfide were highest in the 2013-14 NHANES wave, while metabolites from acrolein, acrylonitrile/vinyl chloride/ethylene oxide, benzene, 1-bromopropane, 
crotonaldehyde, cyanide, N,N-dimethylformamide, and ethylbenzene/ styrene were highest in the 2011-12 NHANES wave.

The proportions of significantly changing metabolites represented in the NHANES waves also changed (Fig. 2). The metabolites HPMMA, 3HPMA, and AMCC were the three most abundant metabolites in the 2005-06 NHANES wave. PGA surpassed AMCC to become the third most abundant metabolite in the 2011-12 and 2013-14 waves. HPMMA and 3HPMA decreased by approximately three percentage points, while PGA, a metabolite of ethylbenzene/styrene, increased by nearly six percentage points relative to other significantly changing UM-VOCs from the 2005-06 NHANES wave. Metabolites of acrolein, acrylamide, carbon-disulfide, crotonaldehyde, and N,N-dimethylformamide were proportionally highest in the 2005-06 NHANES wave, while metabolites of acrylonitrile/ vinyl chloride/ethylene oxide, benzene, 1-bromopropane, and cyanide were proportionally highest in the 2011-12 NHANES wave. Metabolites of acrylonitrile and ethlybenzene/ styrene were proportionally highest in the 2013-14 NHANES wave.

The three largest proportional decreases in UM-VOCs from the 2005-06 to the 2013-14 NHANES waves were observed with metabolites from acrylamide, 1-bromopropane, and acrolein. The proportion of UM-VOCs attributed to acrylamide-GAMA decreased from $1.54 \%$ to $1.02 \%$ (a decrease of $33.5 \%$ ), 1-bromopropane-BPMA decreased from $0.48 \%$ to $0.38 \%$ (a decrease of $21.7 \%$ ), and the proportion of UM-VOCs attributed to acrolein3HPMA decreased from $20.44 \%$ to $17.98 \%$ (a decrease of $12.5 \%$ ). The three largest proportional increases from the 2005-06 to 2013-14 NHANES waves were metabolites from ethylbenzene/styrene, cyanide, and benzene. The proportion of ethylbenzene/styrenePGA increased from $9.57 \%$ to $16.08 \%$ (an increase of $68.1 \%$ ), cyanide-ATCA increased from $7.47 \%$ to $9.94 \%$ (an increase of $33.1 \%$ ), and the proportion of benzene-PMA increased from $0.067 \%$ to $0.069 \%$ (an increase of $4.0 \%$ ).

A cumulative VOC exposure score was computed by adding quintile scores for representative UM-VOCs for each NHANES participant. Quantile regression on these scores showed that in relation to the 2005-06 NHANES wave, all quantiles of the cumulative VOC exposure scores have significantly increased with the middle VOC exposure quantiles showing more dramatic changes than the extremes (Fig. 3).

The cumulative VOC exposure score was then used to identify sociodemographic and potential exposure factors associated with secular trends in VOC exposures. Table 3 describes the mean cumulative VOC exposure score and associated p-values for all sociodemographic factors of interest. Cumulative VOC exposure scores were significantly different across years $(\mathrm{p}<0.0001)$. The 2011-12 NHANES cohort had the highest mean score $(29.85 \pm 0.40$ ), while the 2005-06 NHANES cohort had the lowest mean score (25.37 \pm 0.59 ). Cumulative VOC exposure scores were significantly higher in participants who were older, were female, or who were Mexican American, and in individuals reporting having less than a high school education and being physically inactive. No associations were observed with the cumulative VOC exposure score and participant weight, alcohol consumption, or household income. No significant interactions were observed with year (NHANES wave) and sociodemographic variables in the cumulative VOC exposure 
regression models. However, significant interactions were observed with the potential exposure source variables for participants with private well water and use of dry cleaning. The association between these two potential exposure source variables and VOC levels varied by year (Table S4).

Survey-weighted regression models for individual associations with all $20 \mathrm{UM}-$ VOCs and each sociodemographic factor demonstrated that females have 14 significantly elevated UMVOCs (ranging from $9.33 \%$ to $58.52 \%$ higher) compared with men. Older individuals had elevated levels of 10 UM-VOCs (ranging from $7.33 \%$ to $28.24 \%$ higher) compared with younger individuals. Participants living with three or fewer people in the household had higher levels of 6 UM-VOCs (ranging from $9.49 \%$ to $22.74 \%$ higher) compared with individuals living with more han three people. Race was associated with 6 UM-VOCs. AAMA, ATCA, and 2HPMA were significantly higher in Mexican Americans, while AMCC was elevated in non-Hispanic Whites and TTCA was elevated in Other/Multi-Racial.

Metabolites of acrolein (CEMA), 1,3-butadiene (DHBMA and MHBMA3), and N,Ndimethylformamide (AMCC) had the most significant associations with the sociodemographic factors of interests. All 4 metabolites were significantly higher in individuals who were older. The acrolein and 1,3-butadiene metabolites were significantly higher in individuals who reported being inactive and in participants who lived in a household with three or fewer inhabitants. CEMA and MHBMA3 were higher in individuals who reported consuming fewer than 12 alcoholic beverages in a year, while AMCC was higher in individuals who reported consuming more than 12 alcoholic beverages in a year. CEMA and AMCC were significantly higher in overweight individuals. CEMA and DHBMA were higher in individuals reporting indices of lower socioeconomic status, less than a high school education, and an annual household income of less than $\$ 20,000$. Both 1,3-butadiene metabolites (DHBMA and MHBMA3) and AMCC were significantly higher in females.

We found that VOC metabolites created three latent variables, which indicates three main sources of exposure in our cohort. The path diagram (Fig. 4) shows directed links from factors to variables that are associated with a 0.3 or greater magnitude in loading estimates. The key characteristic metabolites found in factor 1 were 1,3-butadiene, crotonaldehyde, $\mathrm{N}, \mathrm{N}$-dimethylformamide, ethylbenzene/styrene, styrene, toluene, and acrylamide which, explains $57 \%$ of the variance in the data. These VOCs are commonly associated with combustion byproducts including automobile exhaust and consumer products manufacture and use (ATSDR, 2019; Batterman et al., 2014). Factor 2 was distinguished by acrolein, 1brompropane, propylene oxide, and acrylonitrile. Factor 2 explains $36 \%$ of the variance in the data. The VOC in factor 2 are commonly associated with combustion by-products, as well as industrial manufacturing processes (ATSDR, 2019). Factor 3 explains $8 \%$ of the variance in the data. The metabolites extracted by factor 3 were both of the xylene metabolites. Xylene is associated with solvents used in industrial manufacturing processes and is commonly used as a chemical indicator for identifying vehicle exhaust emissions (ATSDR, 2019; Nelson and Quigley, 1983). 


\section{Discussion}

In this study, we examined the relationship between national ambient air VOC levels and urinary metabolites of VOCs from 2005 to 2014. Our analysis showed that in the United States, ambient air VOC levels have continued to decline since 2005, which is consistent with other reports (EPA, 2016a; 2016b; McCarthy et al., 2007). However, secular increases in urinary metabolites of VOCs in participants of NHANES waves 2005-06, 2011-12, and 2013-14 did not support the notion that decreasing trends in national ambient air VOCs would lead to a corresponding decrease in individual-level exposure as assessed by urinary metabolites of VOCs, suggesting that ambient air may not be a major source of VOC exposure.

It has been well established that personal exposure to VOCs is more closely correlated with indoor exposure than with outdoor exposure (Batterman et al., 2014; Meng et al., 2005). However, it was unexpected that ambient concentrations played seemingly very little, if any, role in personal exposure to VOCs, especially given that outdoor VOC sources do partially contribute to indoor VOC levels (Meng et al., 2005). This lack of influence of ambient air VOC concentrations on personal exposure has not been previously shown. While personal exposures typically have a stronger correlation with indoor environments, we expected to see a paralleled influence, albeit maybe small, of the decline in ambient air VOC concentrations on personal exposure. However, we found no association between ambient VOC levels and measurements of personal VOC exposures.

The increases in personal-level exposure to VOCs from NHANES 2005-06 to 2013-14 were observed with all but three metabolites (3MHA \& 4MHA, MA, and 2HPMA), while all national mean ambient air VOC measures decreased from 2005 to 2013. We observed that the contribution of individual VOCs to the composition of significantly changing UM-VOCs has shifted proportionally from the 2005-06 NHANES wave. Age, sex, race, education, and physical inactivity were shown to be associated with higher cumulative VOC exposures, but these characteristics cannot explain the differences between personallevel exposure to VOCs and the secular trends in national ambient air VOC levels.

Previous work has examined ambient air VOC levels from both - a bottom-up approach using emissions inventories and a top-down approach using ambient measures modelling. Global increases in total non-methane VOC emissions have been reported for almost a half a century by bottom-up methodologies such as the Emission Database for Global Atmospheric Research (EDGAR) and other global emissions inventories (Crippa et al., 2018). However, decreases in ambient estimates of VOCs in the United States have been observed for several decades by both bottom-up and top-down approaches (EPA, 2016a; McCarthy et al., 2007). According to the EPA, decreases in VOC emissions from on-road vehicles are the largest source category contributing to the decline in VOCs since 1990. Stricter emissions controls, the implementation of improved technologies, and lower emitting materials have also significantly contributed to the declining ambient VOC trends observed in the United States (EPA, 2016a). Our analysis, which utilized a top-down approach, supports the previous findings of decreases in ambient VOCtrends in the United States. 
Information regarding national-level distributions of personal-level trends in exposure to VOCs over time is lacking. Our analysis is the first quantitative and representative investigation to use urinary metabolites of VOCs to assess secular trends in individual-level exposures. Previous nationally representative analyses have reported decreasing trends in blood VOCs. The decreasing trends in personal concentrations of VOCs observed in previous NHANES analyses using blood VOC measures closely follow the ambient air VOC trends throughout the 1990s. However, blood VOCs do not adequately capture an individuals' daily exposure spectrum to VOCs (Jain, 2017; F.-C. Su et al., 2011).

For estimates of exposure to VOCs, we used urinary metabolites of VOCs. In comparison with blood VOC measurements, the urinary metabolites of VOCs, have fewer kinetic influences and have a relatively delayed clearance, resulting in measurements representative of a broader exposure window: minutes to hours for blood, versus hours to days for urine (Heinrich-Ramm et al., 2000). Consequently, the urinary metabolites of VOCs better reflect day-to-day VOC exposures and are able to capture indoor, personal-care, and volatile chemical products exposures, whose concentrations would quickly diminish in blood biomonitoring measurements. Additionally, a number of federal agencies have been successfully using metabolites as accurate, sensitive, and precise biomarkers of occupational and environmental exposures and their use has been extensively reviewed (DeRooij et al., 1998; Mathias \& B'Hymer, 2014; van Welie et al., 1992). Furthermore, external measures of exposure to VOCs can present significant challenges. It has been shown that personal exposures are often greater than the sum of indoor and outdoor pollution levels (Wallace, 1991); because fully accounting for the numerous routes and sources of exposure is extremely challenging. Biomarker estimates better account for variations in toxicant absorption and metabolism which provide an individuals' internal toxicant dose (DeRooij et al., 1998).

Using urinary metabolites of VOCs as a proxy for VOC exposure, we found that not only was there an increase in VOC exposure from 2005 to 2013, the relative contribution of individual VOCs to personal exposures has shifted. These patterns might reflect changes in exposure sources. The divergence of emissions and ambient air VOCs trends from personal exposures suggests the emerging importance of indoor VOC sources in addition to emission and ambient concentrations. Detailed mass balance analyses conducted by McDonald et al. (2018) have demonstrated changes to the proportions of sources that contribute to anthropogenic VOC emissions. The change in anthropogenic VOC emissions sources in the United States is attributed to the successful control on ambient air pollution. McDonald et al., proposed a shift in the relative importance towards volatile chemical products.

We also found that individual-level exposure to VOCs increased from $0.3 \%$ to $53.6 \%$ per VOC, while ambient air VOCs continued to decrease from 2005 to 2014. These findings suggest that ambient VOC measures are not representative of personal daily exposure to VOCs and/or that ambient VOCs are not the primary source of exposure to VOCs in the general United States population. Moreover, our analysis indicates that both the magnitude and the nature of individual-level exposures to VOCs have changed. Concentrations of all but 3 UM-VOCs increased from 2005-06 to 2013-14, and the contribution of individual 
VOCs to the composition of the mixture of UM-VOC also changed over NHANES waves (2005-06, 2011-12 and 2013-14).

We estimated relative decreases in exposures to crotonaldehyde and acrolein, VOCs predominantly associated with combustion by-products including automobile exhaust and oil/coal burning power plants (ATSDR, 2019). In contrast, relative increases in exposure were observed for ethylbenzene, styrene, and cyanide, which are all VOCs associated with consumer products manufacture and use (ATSDR, 2019). VOCs such as ethylbenzene, styrene, and cyanide, which show increases in both national average concentrations and increases in proportional representation of UM-VOCs mixtures, may indicate an increase in relative importance.

Our results revealed a significant divergence in combustion by-product-associated VOCs from consumer products manufacturing-associated VOCs. In the time range studied, we found a significant reduction in ambient concentrations of carbon-disulfide, acrylonitrile, acrolein, ethylbenzene, styrene, and benzene although there were significant increases in individual exposures to these VOCs, which are chiefly associated with consumer products manufacture and use. Moreover, significant reductions in ambient concentrations of VOCs associated with combustion by-products - toluene, xylene, and 1,3-butadiene were observed with no significant changes in individual exposures to these VOCs, suggesting that reductions in the emissions from combustion sources are not reflected in individual-level exposures. Moreover, the overall decreases in ambient VOC concentrations combined with increases in personal exposures to VOCs associated with consumer products and the lack in significant change in personal exposure to VOCs associated with combustion by-products supports a shift in the relative contribution of VOC exposure sources away from ambient combustion sources and towards volatile chemical products. Although due to the nature of our study, we cannot provide evidence for causative links, we speculate that indoor sources, personal-care products, and volatile chemical products are likely to be major contributors to overall VOC exposure.

Our analysis of the demographic determinants of exposure showed that cumulative VOC exposure scores were significantly higher in older individuals, females, Mexican Americans, in individuals with less than a high school education, as well as those who were less physically active. These findings suggest that VOC exposure may be higher in populations that are generally considered more vulnerable to environmental exposures (Sheiham, 2009). Globally, the disproportionate effects of pollution on the poor and on women have been well documented (Landrigan et al., 2018). The burden of disease on the poor and on women has historically been attributed to indoor domestic activities of more "traditional" household roles. It is also well-established that indoor VOC levels can be significantly higher than outside levels (Wallace, 1991). Disproportionate effects associated with household air pollution coupled with significantly higher VOCs indoors indicate that more time spent indoors could result in higher VOC exposures. Several of the demographic sub-groups identified in our analysis as having higher cumulative VOC exposures likely spend more time inside. Nevertheless, these observations do not help explain the observed secular trends of increased VOC exposures in the United States. 
Occupational exposures could also lead to higher levels of urinary VOC metabolites; however, significant occupational effects on the general population are rarely observed ( $\mathrm{Su}$ et al., 2013). We found that VOCs which are commonly associated with occupational exposures such as toluene, xylene, and styrene had no significant mean changes in concentration in the NHANES participants between 2005 and 2014, an observation that further reinforces the view that occupational exposures do not explain the VOC trends. This view is further supported by our analysis, which showed that in quantile regression models the middle exposure quantiles were changing more rapidly than the extremes. Since occupational exposures are often considered upper-percentile exposures (Su et al., 2013), this increase is not consistent with a secular increase in occupational exposure. Indeed, in the United States there has been a steady decline in industries where solvents use is common, leading to a substantial decline in occupational exposures (Panko et al., 2009; Su et al., 2013). Finally, even though we did not directly quantify occupational exposure, we found no significant relationship between occupational categories and secular cumulative VOC exposures. Taken together, these findings support the notion that occupational exposure cannot explain the secular trends in VOC exposures.

Our study has many strengths. The study covered a large span of time and the data analyzed come from a well-powered cohort representative of the United States. Few studies have assessed secular trends in VOCs in a nationally representative manner. Still fewer have assessed these trends both on an ecological and cross-sectional scale. Hence, the present study seems to be the first to assess secular trends using urinary metabolites as biomarkers of individual-level exposure to VOCs, and the first to compare these trends with ambient VOC trends. The NHANES data are unique and valuable in providing a quantitative multiyear history of population exposures to VOCs in the U.S., and the novel generation of a cumulative VOC exposure score provides unique insight into the nature and the associations of temporal changes in VOC exposure on a national level.

Nonetheless, the study has some limitations. First, all analytical methods of UM-VOC analysis in each NHANES wave were unchanged. However, upon close review of NHANES documentation, we noted the 2005-06 UM-VOC analysis was conducted on residual urine samples (NCHS, 2018). The 2005-06 surplus urine samples were processed and then stored at less than $-20{ }^{\circ} \mathrm{C}$ until samples were pulled for VOC analysis. Urinary metabolites are generally stable, and internal stability studies conducted by NHANES showed no evidence for metabolite degradation. The proper handling of samples, stability of the urinary VOC metabolites, consistency of analytical protocol, and internal validation studies provide a sufficient indication of the validity of the observed concentrations.

Nuances in ambient air monitoring data are also noteworthy. Factors such as meteorology, sampling methodology, and sampling location introduce variability into ambient air measures data. Also, of note in regards to the ambient VOC data- in our study, trends in ambient VOCs were assessed using arithmetic means as opposed to the geometric means used for the urinary metabolites of VOCs. While, arithmetic means are more vulnerable to outliers the EPA uses strict quality control standards for the data summarized in the annual reports (EPA, 2016a). Therefore, the ambient VOC measures should not be unduly influenced by outliers and we are confidents that the arithmetic means adequately reflect 
secular trends in ambient VOCs. For this analysis it is important to note that long-term declines in ambient VOCs across a number of periods are quite consistent and indicate the effectiveness of emission controls (McCarthy et al., 2007).

We were unable to verify whether NHANES participants were in locations with air quality networks. However, given the well-documented national trends of decreasing concentrations of ambient air VOCs we would expect decreases in ambient concentrations of VOCs regardless of where the NHANES participants are living in the country.

Finally, uncertainties arise from the lack of information on actual exposures and individual variation including genetic and physiological differences that could lead to different rates of VOC metabolism. While a relatively lengthy questionnaire about potential VOC exposure sources was administered to each NHANES participant contributing to the UM-VOC data, our analysis found few associations with these potential sources and no significant explanatory variable attributable to the secular changes in UM-VOCs was identified. Nevertheless, there is sufficient warrant to conduct more detailed exposure assessments to identify new potential explanatory variables. Once identified, these data could be added to future studies to aid in the identification of the major contributors of VOC secular trends.

Additional work is also needed to assess VOC exposures geographically and by urban/rural subgroups. It is presently unknown if or to what extent spatial variability exists in personal exposure to VOCs; however, the observed secular trends in exposure to VOCs in the United States, identified in this investigation, are not likely to be solely attributed to this variation. Nevertheless, the increasing proportion of people living in urban environments may be an important contributing factor. Additionally, an analysis of health impacts and economic implications associated with VOC exposures is needed. It is likely that the direct and indirect economic losses accrued over several generations could outweigh the capital investments needed for the implementation of volatile chemical products-reduction initiatives. These analyses would strengthen the rationale for a shift in focus to volatile chemical products and the development of more strict standards for VOC emissions.

In conclusion, the divergence of national personal exposure to VOCs from ambient air VOC concentrations combined with mid-exposure level shifts in the nature of VOC exposures points to the emerging importance of a new sector of VOCs. Our study supports a shift in the relative importance of volatile chemical products on VOC exposures. Adjusting the focus from being solely centered on transportation-derived VOC sources towards the inclusions of volatile chemical products such as personal-care products, adhesives, cleaning agents, coatings, pesticides, and printing inks should be considered when addressing the environmental and health burdens of VOCs.

\section{Supplementary Material}

Refer to Web version on PubMed Central for supplementary material.

\section{Acknowledgments}

This work was supported in part by the NIH grant ES 023716. We thank Jordan Lynch for editorial support. 


\section{References}

Alwis KU, Blount BC, Britt AS, Patel D, Ashley DL, 2012 Simultaneous analysis of 28 urinary VOC metabolites using ultra high performance liquid chromatography coupled with electrospray ionization tandem mass spectrometry (UPLC-ESI/MSMS). Anal. Chim. Acta 750, 152-160. 10.1016/j.aca.2012.04.009. [PubMed: 23062436]

ATSDR Agency for Toxic Substances and Disease Registry, 2017 ATSDR's substance priority list. Retrieved from. https://www.atsdr.cdc.gov/spl/\#2017spl.

ATSDR Agency for Toxic Substances and Disease Registry, 2019 Agency for toxic substances and disease Registry. Retrieved from. https://www.atsdr.cdc.gov.

Batterman S, Su FC, Li S, Mukherjee B, Jia C, Committee HEIHR, 2014 Personal exposure to mixtures of volatile organic compounds: modeling and further analysis of the RIOPA data. Res. Rep. Health Eff. Inst 181, 3-63.

Bell ML, Ebisu K, Peng RD, Samet JM, Dominici F, 2009 Hospital admissions and chemical composition of fine particle air pollution. Am. J. Respir. Crit. Care Med 179 (12), 1115-1120. 10.1164/rccm.200808-1240OC. [PubMed: 19299499]

Benowitz NL, Hukkanen J, Jacob P 3rd, 2009 Nicotine chemistry, metabolism, kinetics and biomarkers. Handb. Exp. Pharmacol 192, 29-60. 10.1007/978-3-540-69248-5_2.

Brook RD, Rajagopalan S, Pope CA 3rd, Brook JR, Bhatnagar A, Diez-Roux AV, 2010 Particulate matter air pollution and cardiovascular disease: an update to the scientific statement from the American Heart Association. Metabolism.. Circulation 121 (21), 2331-2378. 10.1161/ CIR.0b013e3181dbece1. [PubMed: 20458016]

Crippa M, Guizzardi D, Muntean M, Schaaf E, Dentener F, van Aardenne JA, Janssens-Maenhout G, 2018 Gridded emissions of air pollutants for the period 1970-2012 within EDGAR v4.3.2. Earth Syst.Sci. Data 10 (4), 1987-2013. 10.5194/essd-10-1987-2018.

Delgado-Saborit JM, Aquilina NJ, Meddings C, Baker S, Harrison RM, 2009 Model development and validation of personal exposure to volatile organic compound concentrations. Environ. Health Perspect 117 (10), 1571-1579. 10.1289/ehp.0900561 . [PubMed: 20019908]

DeRooij B, Commandeur J, Vermeulen N, 1998 Mercapturic acids as biomarkers of exposure to electrophilic chemicals:applications to environmental and industrial chemicals. Biomarkers 3 (45), 239-303. 10.1080/135475098231101. [PubMed: 23899357]

Di Q, Wang Y, Zanobetti A, Wang Y, Koutrakis P, Choirat C, Schwartz JD, 2017 Air pollution and mortality in the medicare population. N. Engl. J. Med 376 (26),2513-2522. 10.1056/ NEJMoa1702747. [PubMed: 28657878]

Dockery DW, Pope CA 3rd, Xu X, Spengler JD, Ware JH, Fay ME, Speizer FE, 1993 An association between air pollution and mortality in six U.S. cities. N. Engl. J. Med 329 (24), 1753-1759. 10.1056/NEJM199312093292401. [PubMed: 8179653]

EPA U.S. Environmental Protection Agency, 1999 Air method, toxic organics-15 (TO-15): Compendium of methods for the determination of toxic organic compounds in ambient air. Determination of Volatile Organic Compounds (VOCs) in Air Collected in Specially-Prepared Canisters and Analyzed by Gas Chromatography/Mass Spectrometry (GC/MS) EPA 625/ R-96/010b. Retrieved from. https://www.epa.gov/homeland-security-research/epa-air-methodtoxic-organics-15-15-determination-volatile-organicseconded.

EPA U.S. Environmental Protection Agency, 20162014 national emissions inventory report. Retrieved from https://www.epa.gov/air-emissionsinventories/2014-national-emissions-inventory-nei-data .

EPA U.S. Environmental Protection Agency, 2016 Air toxics -urban air toxics monitoring Program. Retrieved from https://www3.epa.gov/ttnamti1/uatm.html .

EPA U.S. Environmental Protection Agency, 2016 National ambient air quality standards (NAAQS) table. Retrieved from https://www.epa.gov/criteria-air-pollutants/naaqs-table .

Franklin M, Koutrakis P, Schwartz P, 2008 The role of particle composition on the association between PM2.5 and mortality. Epidemiology 19 (5), 680-689. [PubMed: 18714438]

Harley RA, Sawyer RF, Milford JB, 1997 Updated photochemical modeling for California's South Coast Air Basin: comparison of chemical mechanisms and motor vehicle emission inventories. Environ. Sci. Technol 31 (10), 2829-2839. 10.1021/es9700562. 
Heinrich-Ramm R, Jakubowski M, Heinzow B, Christensen JM, Olsen E, Hertel O,2000 Biological monitoring for exposure to volatile organic compounds (VOCs). Pure Appl. Chem 72 (3), 385436. 10.1351/pac200072030385.

Jain RB, 2015 Distributions of selected urinary metabolites of volatile organic compounds by age, gender, race/ethnicity, and smoking status in a representative sample of U.S. adults. Environ. Toxicol. Pharmacol 40 (2), 471-479. 10.1016/j.etap.2015.07.018. [PubMed: 26282484]

Jain RB, 2017. Detection rates, trends in and factors affecting observed levels of selected volatile organic compounds in blood among US adolescents and adults. Environ. Toxicol. Pharmacol 56, 21-28. 10.1016/j.etap.2017.08.031. [PubMed: 28869856]

Jerrett M, Burnett RT, Pope CA 3rd, Ito K, Thurston G, Krewski D, Thun M, 2009 Long-term ozone exposure and mortality. N. Engl. J. Med 360 (11),1085-1095. 10.1056/NEJMoa0803894. [PubMed: 19279340]

Jimenez JL, Canagaratna MR, Donahue NM, Prevot AS, Zhang Q, Kroll JH, Worsnop DR, 2009 Evolution of organic aerosols in the atmosphere. Science 326 (5959), 1525-1529. 10.1126/ science.1180353. [PubMed: 20007897]

Laden F, Neas LM, Dockery DW, Schwartz J, 2000 Association of fine particulate matter from different sources with daily mortality in six U.S. cities. Environ. Health Perspect 108 (10), 941947. 10.1289/ehp.00108941. [PubMed: 11049813]

Landrigan PJ, Fuller R, Acosta NJR, Adeyi O, Arnold R, Basu NN, Zhong M,2018 The Lancet Commission on pollution and health. Lancet 391 (10119), 462-512. 10.1016/ S0140-6736(17)32345-0. [PubMed: 29056410]

Mathias PI, B'Hymer C, 2014 A survey of liquid chromatographic-mass spectrometric analysis of mercapturic acid biomarkers in occupational and environmental exposure monitoring. $\mathrm{J}$ Chromatogr B Analyt Technol Biomed Life Sci 964, 136-145. 10.1016/j.jchromb.2014.02.057.

McCarthy MC, Hafner HR, Chinkin LR, Charrier JG, 2007 Temporal variability of selected air toxics in the United States. Atmos. Environ 41 (34), 7180-7194. 10.1016/j.atmosenv.2007.05.037.

McDonaldde Gouw BC, Gilman JB, Jathar SH, Akherati A, Cappa CD, Trainer M, 2018 Volatile chemical products emerging as largest petrochemical source of urban organic emissions. Science 359 (6377), 760-764. 10.1126/science.aaq0524. [PubMed: 29449485]

McDonald BC, Gentner DR, Goldstein AH, Harley RA, 2013 Long-term trends in motor vehicle emissions in u.s. urban areas. Environ. Sci. Technol 47 (17), 10022-10031. 10.1021/es401034z. [PubMed: 23915291]

Meng QY, Turpin BJ, Korn L, Weisel CP, Morandi M, Colome S, ... Maberti S,2005 Influence of ambient (outdoor) sources on residential indoor and personal PM2.5 concentrations: analyses of RIOPA data. J. Expo. Anal. Environ. Epidemiol 15 (1), 17-28. 10.1038/sj.jea.7500378. [PubMed: 15138449]

NCHS (National Center for Health Statistics, 2015 National health and nutrition examination survey home page. Retrieved from. https://www.cdc.gov/nchs/nhanes/index.htm .

NCHS (National Center for Health Statistics, 2018 National Health and Nutrition Examination Survey: Analytic Guidelines, 2011-2012. Retrieved 20 July 2018, from Centers for Disease Control and Prevention, NCHS https://wwwn.cdc.gov/nchs/nhanes/analyticguidelines.aspx .

Nelson PF, Quigley SM, 1983 The m,p-xylenes:ethylbenzene ratio. A technique for estimating hydrocarbon age in ambient atmosphere. Atmos. Environ 17, 659-662. 10.1016/0004-6981(83)90141-5.

Ostro B, Feng WY, Broadwin R, Green S, Lipsett M, 2007 The effects of components of fine particulate air pollution on mortality in California: results from CALFINE. Environ. Health Perspect 115 (1), 13-19. 10.1289/ehp.9281. [PubMed: 17366813]

Ostro BD, Feng WY, Broadwin R, Malig BJ, Green RS, Lipsett MJ, 2008 The impact of components of fine particulate matter on cardiovascular mortality in susceptible subpopulations. Occup. Environ. Med 65 (11), 750-756. 10.1136/oem.2007.036673. [PubMed: 18417555]

Panko JM, Gaffney SH, Burns AM, Unice KM, Kreider ML, Booher LE, Paustenbach DJ, 2009 Occupational exposure to benzene at the ExxonMobil refinery at baton rouge, Louisiana (1977-2005). J. Occup. Environ. Hyg 6 (9), 517-529. 10.1080/15459620903044161. [PubMed: 19544135] 
Peng RD, Bell ML, Geyh AS, McDermott A, Zeger SL, Samet JM, Dominici F,2009 Emergency admissions for cardiovascular and respiratory diseases and the chemical composition of fine particle air pollution. Environ. Health Perspect 117 (6), 957-963. 10.1289/ehp.0800185. [PubMed: 19590690]

Pope CA 3rd, Burnett RT, Thurston GD, Thun MJ, Calle EE, Krewski D, Godleski JJ, 2004 Cardiovascular mortality and long-term exposure to particulate air pollution: epidemiological evidence of general pathophysiological pathways of disease. Circulation 109 (1), 71-77. 10.1161/01.CIR.0000108927.80044.7F. [PubMed: 14676145]

SAS Institute, Inc, 2018 SAS Software 9.4 Copyright @ $2002-2012$. SAS Institute Inc, Cary, NC, USA.

Sheiham A, 2009 Closing the gap in a generation: health equity through action on the social determinants of health. A report of the WHO Commission on Social Determinants of Health (CSDH) 2008. Community Dent. Health 26 (1), 2-3. [PubMed: 19385432]

Su FC, Mukherjee B, Batterman S, 2011 Trends of VOC exposures among a nationally representative sample: analysis of the NHANES 1988 through 2004 data sets. 1994. Atmos. Environ 45 (28), 4858-4867. 10.1016/j.atmosenv.2011.06.016.

Su FC, Mukherjee B, Batterman S, 2013 Determinants of personal, indoor and outdoor VOC concentrations: an analysis of the RIOPA data. Environ. Res 126, 192-203. 10.1016/ j.envres.2013.08.005. [PubMed: 24034784]

van Welie RT, van Dijck RG, Vermeulen NP, van Sittert NJ, 1992 Mercapturic acids, protein adducts, and DNA adducts as biomarkers of electrophilic chemicals. Crit. Rev. Toxicol 22 (5-6), 271-306. 10.3109/10408449209146310. [PubMed: 1489508]

Wallace LA, 1991 Personal exposure to 25 volatile organic compounds. EPA's 1987 team study in Los Angeles, California. Toxicol. Ind. Health 7 (5-6), 203-208. [PubMed: 1780860]

Wallace LA, Pellizzari ED, Hartwell TD, Sparacino C, Whitmore R, Sheldon L, Perritt R, 1987 The TEAM (Total Exposure Assessment Methodology) Study: personal exposures to toxic substances in air, drinking water, and breath of 400 residents of New Jersey, North Carolina, and North Dakota. Environ. Res 43 (2), 290-307. [PubMed: 3608934]

WHO (World Health Organization), 2018 Air pollution. Retrieved from. https://www.who.int/newsroom/fact-sheets/detail/ambient-(outdoor)-air-quality-and-health .

WHO (World Health Organization), 2016 Burden of disease from the joint effects of household and ambient air pollution for 2012- description of method. Retrieved from. www.who.int/phe/ health_topics/outdoorair/databases/AP_jointeffect_BoD_results_Nov2016.pdf .

Zanobetti A, Franklin M, Koutrakis P, Schwartz J, 2009 Fine particulate air pollution and its components in association with cause-specific emergency admissions. Environ. Health 8, 58 10.1186/1476-069X-8-58. [PubMed: 20025755] 




Fig. 1. Percent change in ambient volatile organic compounds and urinary metabolites of volatile organic compounds.

National arithmetic mean percent change for 11 ambient VOCs as reported by the yearly summary statistics for VOC monitoring in the National Monitoring Program (NMP) annual reports from 2005 and to 2013 and geometric mean percent change for 14 corresponding UM-VOCs from 2005-06 to 2013-14 NHANES waves. NHANES = National Health and Nutrition Examination Survey; A, VC, EO = Acrylonitrile, vinyl chloride, ethylene oxide; $\mathrm{VOC}=$ volatile organic compound $\mathrm{UM}-\mathrm{VOC}=$ urinary metabolite of VOC. 

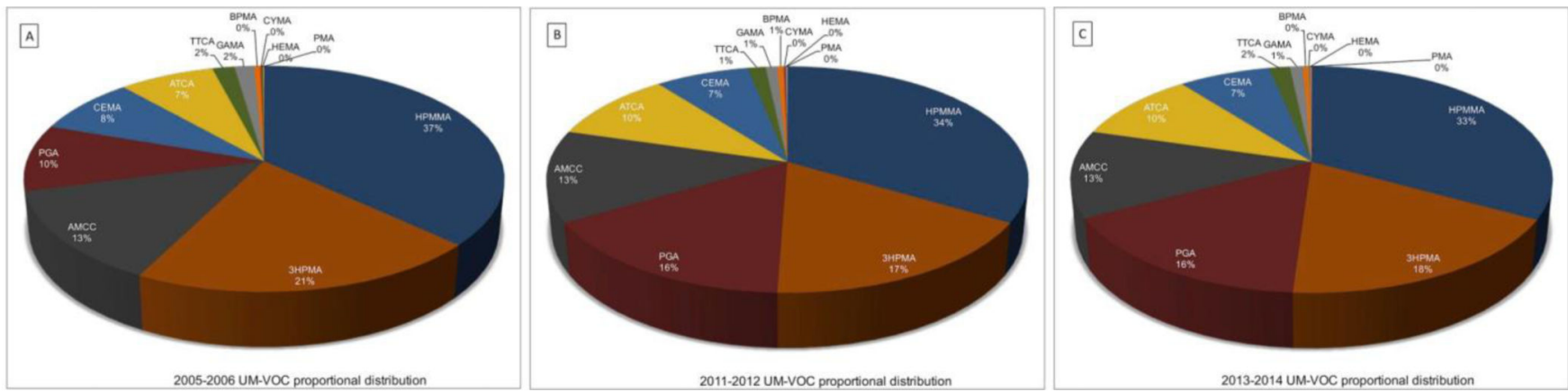

Fig. 2. Proportion of UM-VOCs attributed to each metabolite by NHANES wave.

Proportion of select UM-VOCs attributed to each metabolite by NHANES wave, (A) 200506, (B) 2011-12 and (C) 2013-14. The UM-VOCs represented are metabolite which are significantly changing across the NHANES waves. NHANES $=$ National Health and Nutrition Examination Survey; UM-VOC = urinary metabolite of volatile organic compound. 

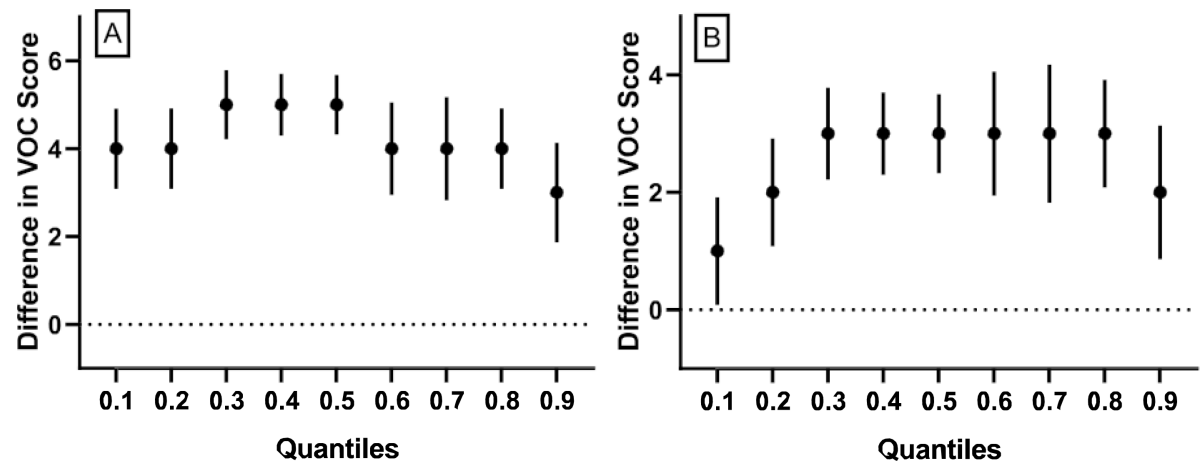

Fig. 3. Quantile regression models of cumulative VOC exposure regressed by NHANES wave. Quantile plot for associations between cumulative VOC exposure score differences by NHANES wave where the 2005-06 NHANES wave is the referent group. The y-axes represent the cumulative VOC exposure score difference. Estimate and $95 \%$ confidence intervals are shown for $0.1,0.2,0.3,0.4,0.50 .6,0.7,0.8$ and 0.9 quantiles for NHANES waves (A) 2011-12 vs. 2005-06 and (B) 2013-14 vs. 2005-06. VOC= volatile organic compound; NHANES = National Health and Nutrition Examination Survey 


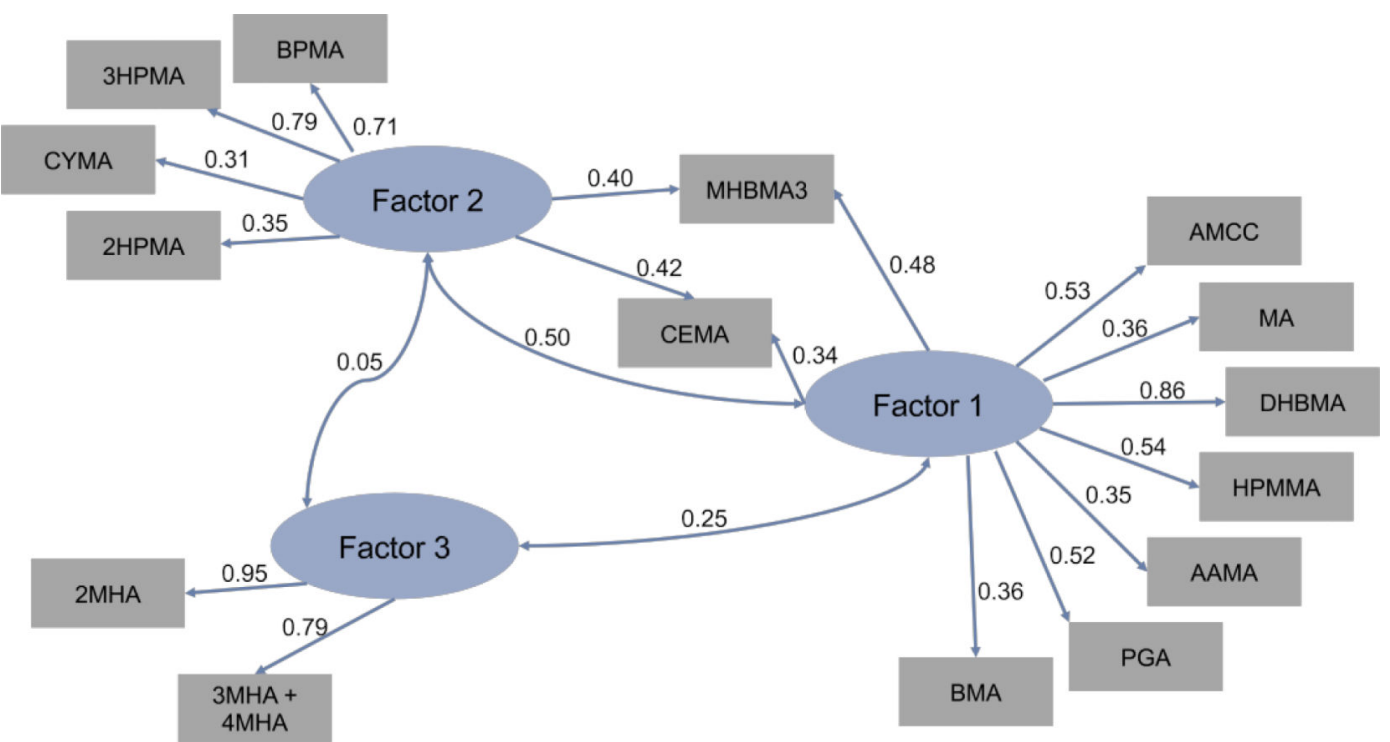

Fig. 4. Factor analysis path diagram of NHANES urinary metabolites of volatile organic compounds.

Factor analysis of creatinine normalized log transformed UM-VOCs. Loading estimates > 0.3 are indicated by the directed links from factors to variables. The double-headed links show correlations between factors. NHANES $=$ National Health and Nutrition Examination Survey; UM-VOC = urinary metabolite of volatile organic compound. 
을

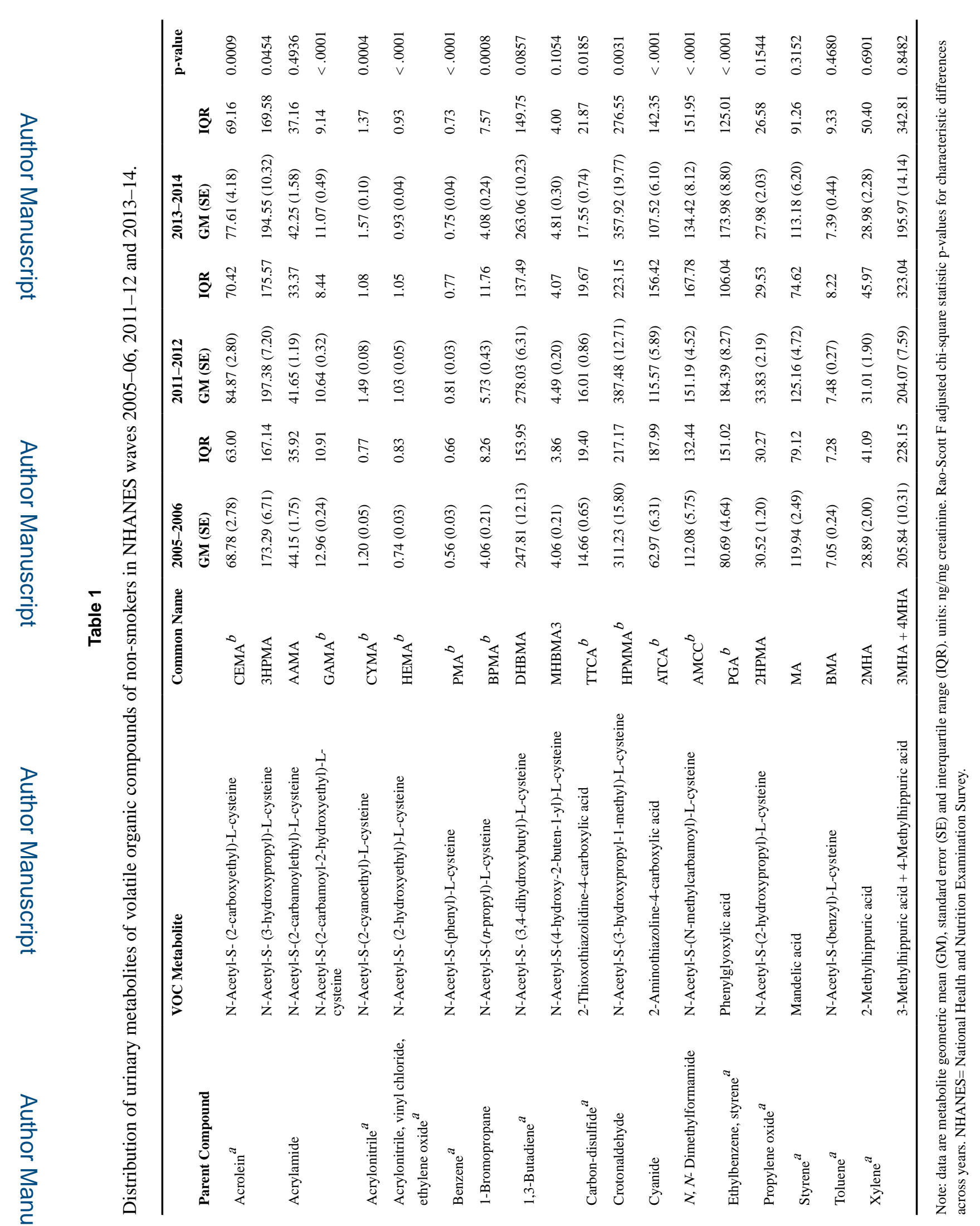

Environ Res. Author manuscript; available in PMC 2020 June 15. 

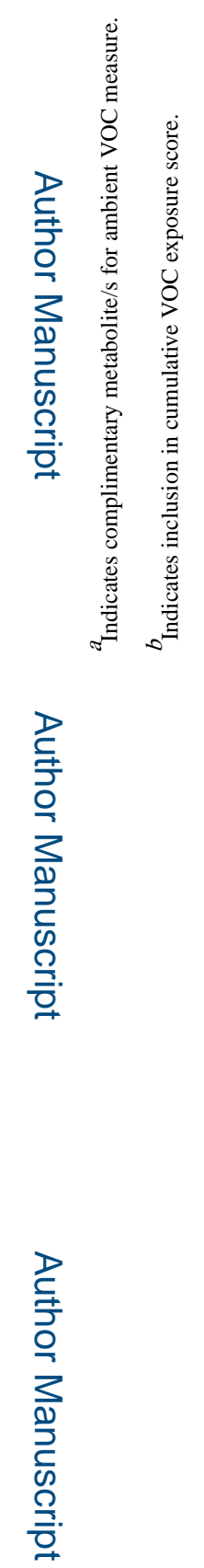

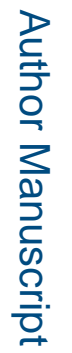

Environ Res. Author manuscript; available in PMC 2020 June 15. 


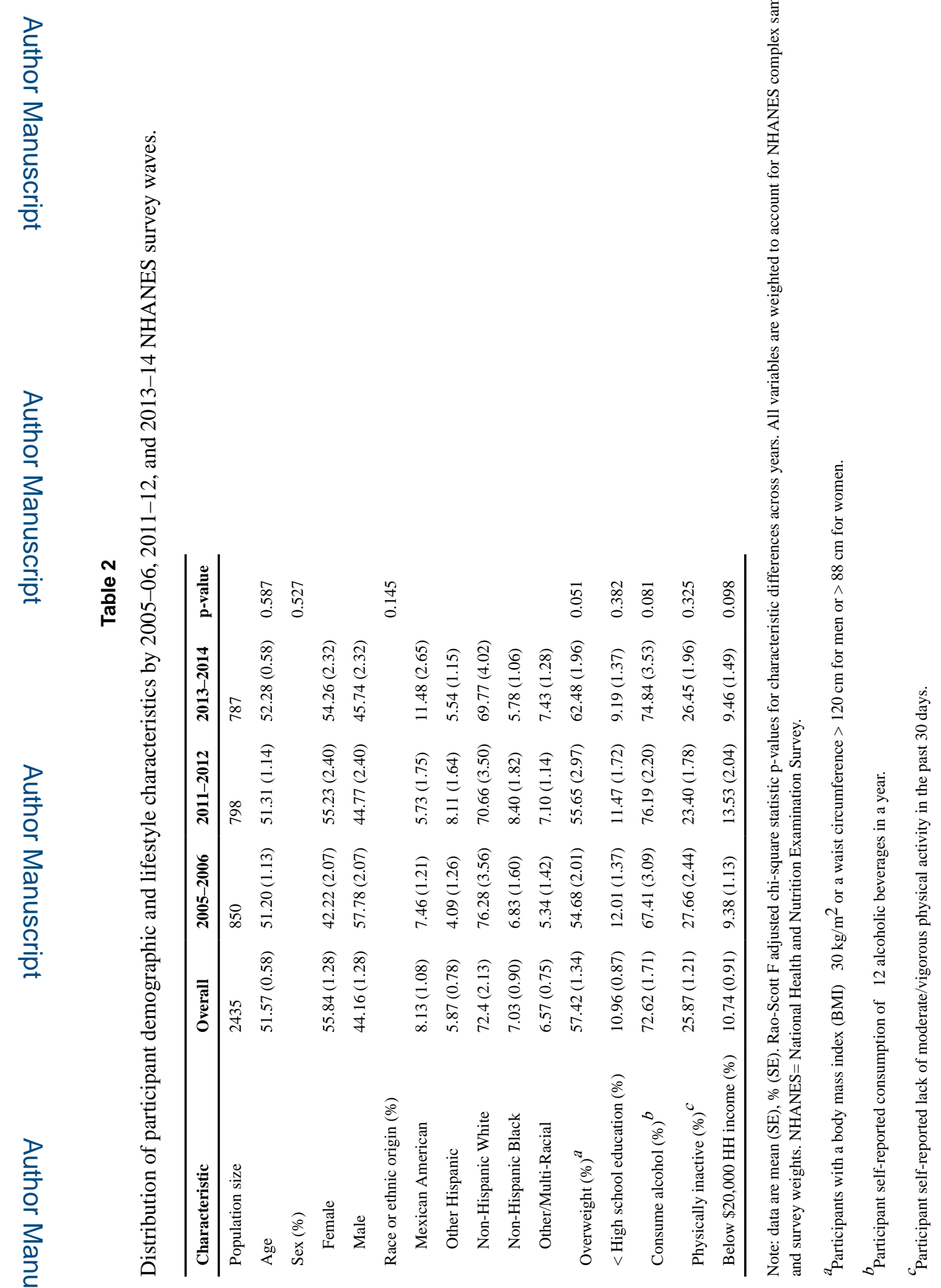

Environ Res. Author manuscript; available in PMC 2020 June 15. 


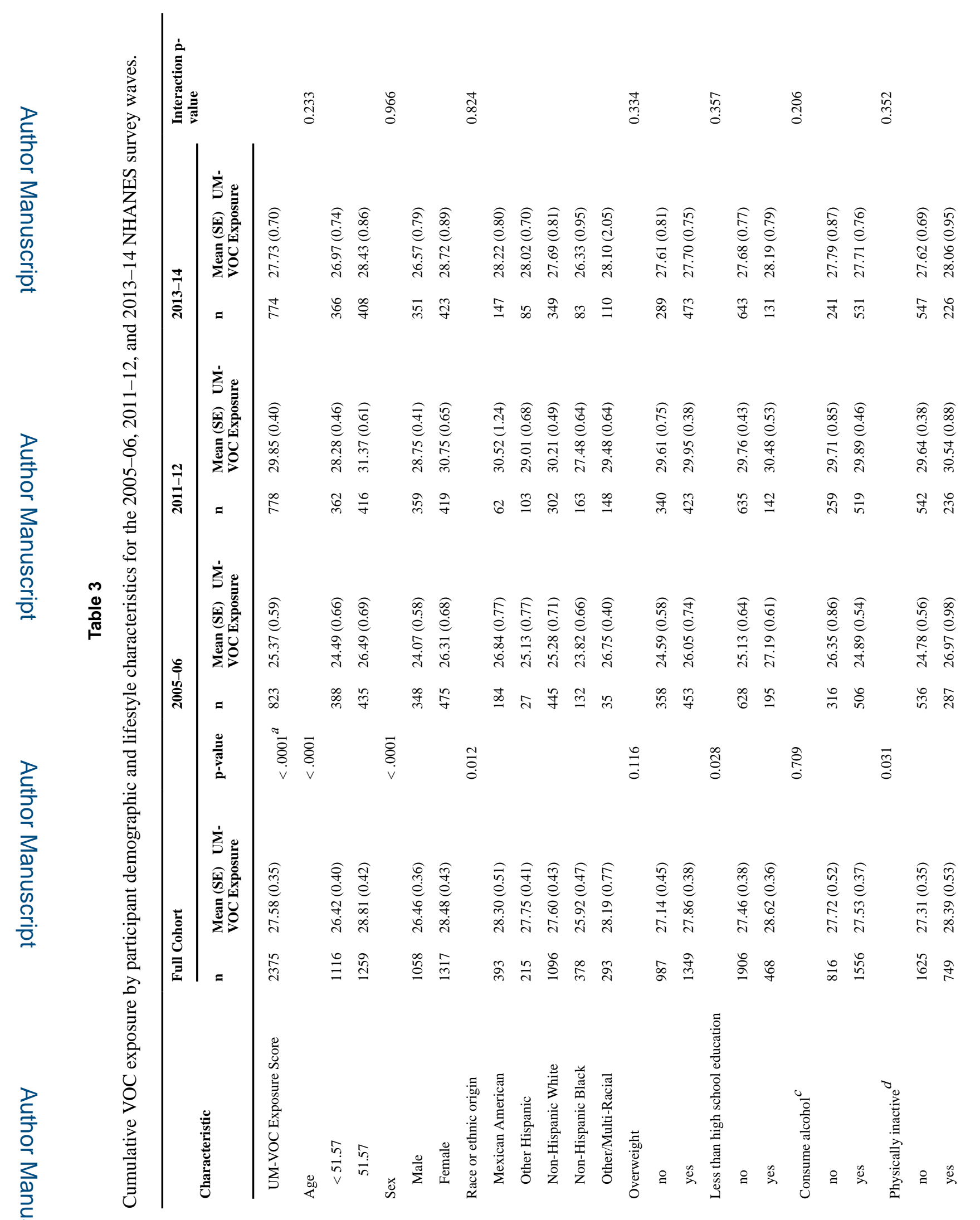

Environ Res. Author manuscript; available in PMC 2020 June 15. 
Konkle et al.

Page 25

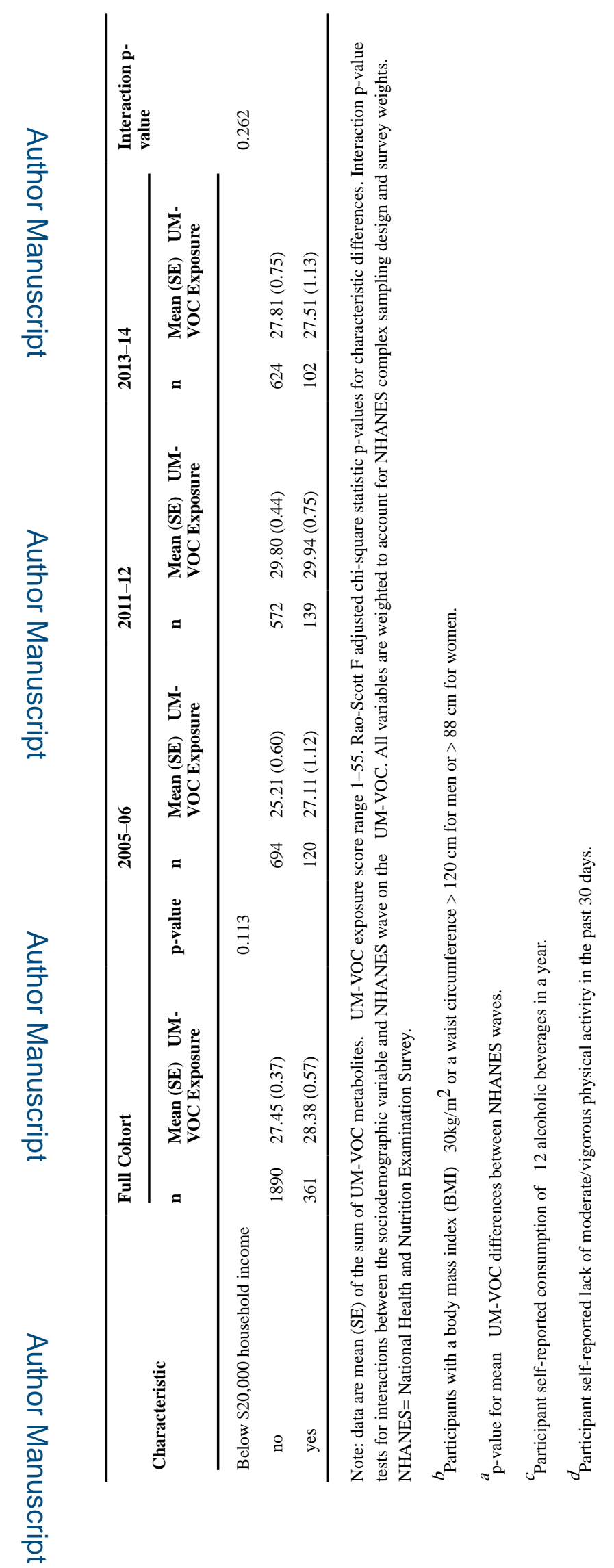

Environ Res. Author manuscript; available in PMC 2020 June 15. 